Pacific Journal of Mathematics

ALMOST ISOMETRIES OF BANACH SPACES AND MODULI 


\section{ALMOST ISOMETRIES OF BANACH SPACES AND MODULI OF PLANAR DOMAINS}

\section{RICHARD ROCHBERG}

Let $C(X)$ and $C(Y)$ be the supremum normed Banach spaces of continuous complex valued functions on the compact Hausdorff spaces $X$ and $Y$ respectively. Let $A$ and $B$ be closed subspaces of $C(X)$ and $C(Y)$ respectively. A map from $A$ to $B$ will mean a continuous invertible linear map of $A$ to $B$. The set of all such maps will be denoted by $L(A, B)$. For $T$ in $L(A, B)$ define $c(T)=1 /\left(\|T\|\left\|T^{-1}\right\|\right)$. A generalization of the Banach-Stone theorem is proved which shows that there is a constant $d<1$ such that if $A$ and $B$ satisfy certain additional technical restrictions and there is a $T$ in $L(A, B)$ with $c(T)>d$ then $X$ and $Y$ are homeomorphic. Furthermore, $T$ is, roughly, composition with this homeomorphism.

For $S$ a connected subset of $C$ bounded by a finite number of disjoint Jordan curves, denote by $A(S)$ the Banach space of functions in $C(S)$ which are analytic on the interior of $S$. For two such domains, $S$ and $S^{\prime}$, set $d\left(S, S^{\prime}\right)=\inf \{-\log c(T) ; T$ a linear map of $A(S)$ onto $A\left(S^{\prime}\right)$ \}. By analyzing maps $T$ for which $c(T)$ is nearly one, it is shown that $d(\cdot, \cdot)$ is a metric on the space of moduli of such domain (considered as Riemann surfaces) and that this metric induces the classical moduli topology.

If $T$ in $L(A, B)$ preserves norms, i.e., if $\|T f\|=\|f\|$ for all $f$ in $A, T$ is called an isometry. If there is an isometry between $A$ and $B$ then $A$ and $B$ are called isometric. If $T$ is of norm one then $c(T)$ is the largest constant such that $c(T)\|f\| \leqq\|T f\| \leqq\|f\|$. It is immediate that $0<c(T) \leqq 1$ for all $T$ in $L(A, B)$ and that $T /\|T\|$ is an isometry if and only if $c(T)=1$. Maps, $T$, for which $c(T)$ is nearly one will be called almost isometries.

Using this notation, the Banach-Stone theorem can be stated as follows.

Theorem. If there is a $T$ in $L(C(X)), C(Y))$ with $c(T)=1$ then $X$ and $Y$ are homeomorphic. Furthermore, any such $T$ is of the form $T f=g \cdot f \circ h$ where $h$ is a homeomorphism from $Y$ to $X$ and $g$ is a continuous function on $Y$ of constant modulus.

More recently, Cambern ([2], [3]) has extended this result to

THEOREM. If there is a $T$ in $L(C(X), C(Y))$ with $c(T)>1 / 2$ then $X$ and $Y$ are homeomorphic.

In $\S 2$ we show that if $A$ and $B$ are closed subspaces (not necessarily subalgebras) of $C(X)$ and $C(Y)$ respectively which satisfy certain 
additional technical restrictions then the following is true.

THEOREM A. There is a constant $d<1$ so that if $A$ and $B$ are allowable subspaces of $C(X)$ and $C(Y)$ respectively and if there is a $T$ in $L(A, B)$ with $c(T)>d$, then $X$ and $Y$ are homeomorphic. Furthermore, there is a function $\varepsilon(x)$ which decreases continuously to zero as $x$ increases to one so that given such a $T$ there is a homeomorphism $h$ of $Y$ to $X$ and a function $g$ of constant modulus so that for all $f$ in $A,\|T f-g \cdot f \circ h\| \leqq \varepsilon(c(T))\|f\|$.

If $A$ and $B$ are any two Banach spaces then the quantity $D(A, B)=\inf \{-\log c(T) ; T$ in $L(A, B)\}$ is a measure of how close $A$ and $B$ are to being isometric. Define $D(A, B)=\infty$ when $L(A, B)$ is empty. This function was first studied by Banach and Mazur ([1]) who observed that it is symmetric, positive semi-definite and satisfies the triangle inequality. Furthermore, if $A$ and $B$ are isometric then $D(A, B)=0$.

This function, applied to certain algebras of analytic functions on planar Riemann surfaces, will be shown to define a metric on the moduli space of those surfaces. Specifically, we denote by $\mathscr{S}$ the set of conformal equivalence classes of Riemann surfaces realizable as connected subsets of the complex plane bounded by two or more (but a finite number of) disjoint Jordan curves. For $n \geqq 2$ denote by $\mathscr{S}_{n}$ the class of surfaces in $\mathscr{S}$ with $n$ boundary contours. For any $S$ in $\mathscr{S}$ we define $A(S)$ to be the subalgebra of $C(S)$ consisting of all functions in $C(S)$ which are analytic on the interior of $S$. It is known that this definition is independent of the particular realization of $S$. For $S$ and $S^{\prime}$ in $\mathscr{S}$, define $d\left(S, S^{\prime}\right)=D\left(A(S), A\left(S^{\prime}\right)\right)$.

In $\S 3$ we introduce a particular set of classical moduli for the sets $\mathscr{S}_{n}$.

In $\S 4$ we introduce a set of conformal invariants for Riemann surface in $\mathscr{S}$ and study the relationship between these invariants and the function $d(\cdot, \cdot)$.

Sections 5 and 6 contain the major parts of the proof of the following.

THEOREM B. For any integer $n \geqq 2, d(\cdot, \cdot)$ is a metric on the space $\mathscr{S}_{n}$. This metric induces the same topology as the classical moduli topology.

A significant portion of the arguments use specific realizations of the Riemann surfaces in question as subsets of the complex plane. It is not clear to what extent these result can be extended to nonplanar surfaces or to surfaces of infinite connectivity. (Some results 
for nonplanar surfaces are presented in [7].)

These results are new, however, results very similar in spirit have been obtained by Nakai ([5]) using different methods. He shows that for a certain class of Riemann surfaces, the extent to which the Royden algebras of two surfaces are almost isometric is directly related to the minimal dilitation of quasiconformal maps between the two surfaces.

In addition to the notation previously introduced and that introduced in the individual sections we will use the following notation.

If $a$ is an element of $C(X)$ and $K$ is a subset of $X$, we set $\|a\|_{K}=\sup \{|f(x)| ; x$ in $K\}$. We will use this notation even though $\|\cdot\|_{K}$ may not be a norm.

Let $D$ be the unit disk of the complex plane. For $z$ and $z^{\prime}$ in $D$ we define $\delta\left(z, z^{\prime}\right)=\left|\left(z-z^{\prime}\right) /\left(1-\bar{z} z^{\prime}\right)\right|$. If $S$ is a Riemann surface which is conformally equivalent to $D$ and $w$ and $w^{\prime}$ are any two points of $S$, we set $\delta_{S}\left(w, w^{\prime}\right)=\delta\left(t(w), t\left(w^{\prime}\right)\right)$ where $t$ is a conformal map of $S$ onto $D$. It is well known that this last definition is independent of the choice of $t$.

2. Properties of almost isometries of Banach spaces. When working with an invertible linear map, $T$, between the Banach spaces we will often use the following notational convention. The spaces will be denoted by two capital Latin letters. Elements of the spaces will be denoted by the corresponding lower case letters. Finally, elements of the spaces will be individuated so that elements with corresponding individuation marks will be elements that correspond under $T$. For example, given $T$ in $L(A, B)$, without further mention our convention guarantees that $a, a^{\prime}$, and $a_{3}$ are in $A ; b, b^{\prime}$, and $b^{\prime \prime}$ are in $B$; and $T(a)=b, T\left(a_{3}\right)=b_{3}$, etc.

Given an element $a$ in the Banach space $C(X)$ and a point $x$ in $X$ we will say that $a$ peaks at $x$ if $a$ attains its maximum modulus at $x$, i.e., $|a(x)|=\|\alpha\|$. We will say that $a$ peaks only at $x$ if $x$ is the only point of $X$ at which $a$ peaks, i.e., $\left|a\left(x^{\prime}\right)\right|<\|a\|$ for all $x^{\prime}$ in $X, x^{\prime} \neq x$. We will say that a sequence of functions $a_{1}, a_{2}, \ldots$ in $C(X)$ is a fundamental sequence at $x$ if

(a) all of the $a_{i}$ are of norm one and peak only at $x$, and

(b) for any $y$ in $X, y \neq x$, the sequence of numbers $\left|a_{1}(y)\right|$, $\left|a_{2}(y)\right|, \cdots$ converges monotonically to zero.

It follows from this definition that if $a_{1}, a_{2}, \ldots$ is a fundamental sequence at $x$ then the $a_{n}$ converge uniformly to zero off every open neighborhood of $x$ and that for any $k$ between zero and one the set on which $\left|a_{n}(y)\right|>k$ shrinks to $x$ as $n$ becomes infinite. Let $A$ be a closed subspace of $C(X)$. We will call $A$ an allowable subspace if for every $x$ in $X$, one can find in $A$ a fundamental sequence at $x$. 
We have not required that an allowable subspace be a subalgebra. It follows from the definition that a closed subalgebra of $C(X)$ is an allowable subspace of $C(X)$ if and only if for any $x$ in $X$ there is an $a$ in $A$ which peaks only at $x$ (i.e., every point of $X$ is a peak point for $A$ ). If $S$ is a finite bordered Riemann surface and $A(S)$ is the algebra of functions continuous on the bordered surface and analytic on the interior of $S$, then $A(S)$, regarded as a subalgebra of $C(\partial S)$, satisfies this condition and hence is an allowable subspace of $C(\partial S)$.

For the remainder of this section we will be considering the following situation. $X$ and $Y$ will be compact Hausdorff spaces and $A$ and $B$ will be allowable subspaces of $C(X)$ and $C(Y)$ respectively. $T$ will be an element of $L(A, B)$ of norm one. We set $c=c(T)=$ $\left\|T^{-1}\right\|^{-1}$. Our goal will be to develop properties of $T$ which follows from the assumption that $c$ is sufficiently near one.

If $c=1$ then some of the proofs in this section are not valid. However, in that case, the results remain valid and are simply known results and their direct corollaries.

2.1. We begin by showing that if $c$ is large enough then, given $x$ in $X$, there is a $y$ in $Y$ so that if $a$ in $A$ peaks at $x$ then $b=T a$ "almost" peaks at $y$.

THEOREM. There is a $c_{0}<1$ such that if $c>c_{0}$ then given $x_{0}$ in $X$ there is a unique $y_{0}$ in $Y$ so that if a peaks at $x_{0}$, then $\left|b\left(y_{0}\right)\right| \geqq$ $(2 c-1)\|a\|$, and hence $\left|b\left(y_{0}\right)\right| \geqq(2 c-1)\|b\|$.

Proof. We begin with a lemma.

Lemma. Given $a_{1}, a_{2}$ in $A$, if $\left|a_{1}(x)\right| \geqq\left|a_{2}(x)\right|$ for all $x$ in $X$ and if $b_{2}$ peaks at $y$, then $\left|b_{1}(y)\right| \geqq c\left\|a_{2}\right\|-((1-c) / c)\left\|a_{1}\right\|$.

Proof of lemma. The result is immediate if $b_{1}$ peaks at $y$. Assume this is not the case. Given $\varepsilon$ positive, pick $U$ an open neighborhood of $y$ so that $\left|b_{1}(w)-b_{1}(y)\right|<\varepsilon$ for all $w$ in $U$. Pick $b_{3}$ so that $\left\|b_{3}\right\|=\left\|b_{1}\right\|-\left|b_{1}(y)\right|$, arg $\left(b_{3}(y)\right)=\arg \left(b_{2}(y)\right), b_{3}$ peaks only at $y$, and $\left|b_{3}\right|<\varepsilon$ off $U$. Hence, for appropriate $\theta$, since $b_{2}$ peaks at $y$,

$$
\begin{aligned}
c\left(\left\|b_{2}\right\|+\left\|b_{3}\right\|\right) & =c\left(\left\|b_{2}+b_{3}\right\|\right) \leqq c\left(\left\|a_{2}+a_{3}\right\|\right) \\
& \leqq c\left(\left\|e^{i \theta} a_{1}+a_{3}\right\|\right) \leqq\left\|e^{i \theta} b_{1}+b_{3}\right\| .
\end{aligned}
$$

But, by the construction of $b_{3}$,

hence

$$
\left\|e^{i \theta} b_{1}+b_{3}\right\| \leqq\left\|b_{1}\right\|+\varepsilon
$$

$$
c|| b_{2}\|\leqq\| b_{1}\|+\varepsilon-c\| b_{3}\|\leqq\| b_{1} \|+\varepsilon-c\left(\left\|b_{1}\right\|-\left|b_{1}(y)\right|\right) .
$$


Since $\varepsilon$ was arbitrary we have

$$
\left|b_{1}(y)\right| \geqq\left\|b_{2}\right\|-\left(\frac{1-c}{c}\right)\left\|b_{1}\right\| \geqq c\left\|a_{2}\right\|-\left(\frac{1-c}{c}\right)\left\|a_{1}\right\| \text {. }
$$

Proof of theorem. For any $b$ in $B$ we define $P_{b}=\{y$ in $Y:|b(y)| \geqq$ $(4 c-3)\|b\|\}$. Pick $a_{1}, \cdots, a_{n}, \cdots$ a fundamental sequence at $x_{0}$. We can assume $a_{n}\left(x_{0}\right)=1$. Let $Q_{\infty}=\bigcap_{n=1}^{\infty} P_{b_{n}}$. $Q_{\infty}$ is the required point $y_{0}$. We prove this by verifying a series of claims.

Claim A. $Q_{\infty}$ is not empty. The $P_{b_{n}}$ are closed subsets of the compact set $Y$. Hence it suffices to show that they have the finite intersection property. Given $N$, we must show that $Q_{N}=\bigcap_{n=1}^{N} P_{b_{n}}$ is not empty. Pick $y$ in $Y$ so that $b_{N}$ peaks at $y$. Since $a_{N}$ is of norm one, $\left|b_{N}(y)\right| \geqq c$. By construction $y$ is in $P_{b_{N}}$. Since the $a_{n}$ form a fundamental sequence, the previous lemma can be applied. For $k<N,\left|b_{k}(y)\right| \geqq c\left\|a_{N}\right\|-((1-c) / c)\left\|a_{k}\right\|=c+1-1 / c$. Hence, if $c$ is greater than $1 / 3$ we have $\left|b_{k}(y)\right| \geqq 4 c-3$. So $y$ is in $P_{b_{k}}$. Hence $y$ is in $Q_{N}$, and thus $Q_{\infty} \neq \varnothing$.

Claim B. $Q_{\infty}$ is a single point. Suppose $Q_{\infty}$ contains two distinct points $y^{\prime}$ and $y^{\prime \prime}$. Choose a small positive $\varepsilon$. Choose $b^{\prime}$ and $b^{\prime \prime}$ from fundamental sequences at $y^{\prime}$ and $y^{\prime \prime}$ so that $\sup _{\theta}\left\|b^{\prime}+e^{i \theta} b^{\prime \prime}\right\| \leqq 1+\varepsilon$. Since $b^{\prime}$ peaks in $Q_{\infty}$, for each $n$ we can find a $\theta$ so that $4 c-2 \leqq$ $\left\|b_{n}+e^{i \theta} b^{\prime}\right\|$. Hence $4 c-2 \leqq\left\|a_{n}+e^{i \theta} a^{\prime}\right\|$. Let $x^{\prime}$ be any point at which $\left|a_{n}\left(x^{\prime}\right)+e^{i \theta} a^{\prime}\left(x^{\prime}\right)\right| \geqq 4 c-2$. We must have $\left|a^{\prime}\left(x^{\prime}\right)\right| \geqq 4 c-3$ and $\left|a_{n}\left(x^{\prime}\right)\right| \geqq 4 c-2-\left|a^{\prime}\left(x^{\prime}\right)\right| \geqq 4 c-2-1 / c$. Pick $c$ so large that for some positive $\varepsilon^{\prime}$ we have

$$
4 c-2-1 / c \geqq \varepsilon^{\prime}>0 \text {. }
$$

Hence $a^{\prime}$ takes a value of modulus greater than or equal to $4 c-3$ at a point $x^{\prime}$ where the modulus of $a_{n}$ is greater than $\varepsilon^{\prime}$. The $a_{n}$ are a fundamental sequence at $x_{0}$. Letting $n$ go to infinity, the set on which $a_{n}$ is of modulus greater than $\varepsilon^{\prime}$ shrinks to the point $x_{0}$ and thus the points $x^{\prime}$ which were chosen depending on $n$ converge to $x_{0}$. By continuity of $a^{\prime},\left|a^{\prime}\left(x_{0}\right)\right| \geqq 4 c-3$. Similarly, $\left|a^{\prime \prime}\left(x_{0}\right)\right| \geqq 4 c-3$. So, for some $\theta$

$$
\left\|a^{\prime}+e^{i \theta} a^{\prime \prime}\right\| \geqq\left|a^{\prime}\left(x_{0}\right)\right|+\left|a^{\prime \prime}\left(x_{0}\right)\right| \geqq 2(4 c-3)=8 c-6 .
$$

So

$$
8 c-6 \leqq \sup \left\|a^{\prime}+e^{i \theta} a^{\prime \prime}\right\| \leqq \frac{1}{c} \sup \left\|b^{\prime}+e^{i \theta} b^{\prime \prime}\right\|<\frac{1}{c}(1+\varepsilon) .
$$

Since $\varepsilon$ was arbitrary we must have $1 / c \geqq 8 c-6$. This is impossible 
if $c>$.89. This contradiction shows that $Q_{\infty}$ is a single point. Denote the point by $y_{0}$.

Claim C. If $\left|b_{N}(y)\right|>2 c-1$ then $\left|b_{k}(y)\right|>4 c-3$ for all $k$ less than $N$. Assume $\left|b_{N}(w)\right|>2 c-1$ and $\left|b_{k}(w)\right|<4 c-3$ for some $w$ and some $k$ less than $N$. Pick $b^{\prime}$ peaking at $w,\left|b^{\prime}(w)\right|=c, \arg b^{\prime}(w)=$ $\arg b_{Y}(w)$, and modulus of $b^{\prime}$ less than some preassigned $\varepsilon$ on $P_{b_{k}}$. (The assumption implies that $w$ is not in $P_{b_{k}}$.) Hence $\left\|b_{N}+b^{\prime}\right\| \geqq 2 c$, and $\sup _{o}\left\|b_{k}+e^{i \theta} b^{\prime}\right\| \leqq 4 c-2$. So, $2 c \leqq\left\|a_{N}+a^{\prime}\right\|$. Let $x$ be a point at which $2 c \leqq\left|a_{Y}(x)\right|+\left|a^{\prime}(x)\right|$. So

$$
\begin{aligned}
4 c-2 \geqq \sup _{\theta}\left\|b_{k}+e^{i \theta} b^{\prime}\right\| & \geqq c \sup _{\theta}\left\|a_{k}+e^{i \theta} a^{\prime}\right\| \\
& \geqq c\left(\left|a_{k}(x)\right|+\left|a^{\prime}(x)\right|\right) .
\end{aligned}
$$

The $a_{n}$ are a fundamental sequence, hence $\left|a_{N}(x)\right| \leqq\left|a_{k}(x)\right|$. So, $4 c-$ $2 \geqq c\left(\left|a_{N}(x)\right|+\left|a^{\prime}(x)\right|\right) \geqq c \cdot 2 c$ which is impossible if $c \neq 1$.

Claim D. Given an open set $U$ in $Y$ with $y_{0}$ in $U$, then for all but finitely many $n,\left\|b_{n}\right\|_{Y-U}<2 c-1$. If not, then there are integers $n_{i}$ increasing to infinity and points $y_{i}$ in $Y-U$ with $\left|b_{n_{i}}\left(y_{i}\right)\right| \geqq 2 c-1$. Let $y^{\prime}$ be an accumulation point of the $y_{i}$. By Claim C, $\left|b_{n}\left(y_{i}\right)\right| \geqq$ $4 c-3$ for $n \leqq n_{i}$. Hence, for each $n$, by the continuity of $b_{n}$, $\left|b_{n}\left(y^{\prime}\right)\right| \geqq 4 c-3$. Thus $y^{\prime}$ is in $Q_{\infty} \subseteq U$. But $y^{\prime}$ is not in $U$. This contradiction establishes the claim.

Claim E. If $a^{\prime}$ is in $A$ and $\left\|a^{\prime}\right\|=a^{\prime}\left(x_{0}\right)=1$ then $\left|b^{\prime}\left(y_{0}\right)\right| \geqq$ $2 c-1$. If not, then there is an open set $U$ in $Y$ containing $y_{0}$ with $\left|b^{\prime}\right| \leqq 2 c-1-\varepsilon$ on $U$. Claim $D$ shows that if we take $n$ large enough we can insure that $\left|b_{n}(y)\right|<2 c-1-\varepsilon$ for $y$ not in $U$. We know that $\left\|a_{n}+a^{\prime}\right\|=2$. Hence $2 c \leqq\left\|b_{n}+b^{\prime}\right\| \leqq \max \left(\left\|b_{n}+b^{\prime}\right\|_{U}\right.$, $\left.\left\|b_{n}+b^{\prime}\right\|_{Y-U}\right) \leqq 2 c-\varepsilon$. This contradiction establishes Claim E.

Claim $\mathrm{E}$ combined with Claim $\mathrm{B}$ shows that the point $y_{0}$ is unique and independent of the original choices of the function $a$ and the fundamental sequence. The theorem is proved.

Definition. For any $x_{0}$ in $X$ let $t\left(x_{0}\right)$ be the point $y_{0}$ in $Y$ such that the previous theorem is satisfied.

During the proof a number of restrictions were placed on $c$. The most severe of these was (2.1). This inequality will be satisfied if $c>c_{0}$ where $c_{0}=(3+\sqrt{17}) / 8=.8904$. There is no reason to assume that this is the best value for which the theorem is true. The results of Cambern referred to earlier are valid if $c>1 / 2$. It may be that $c_{0}=1 / 2$ is also adequate for Theorem A.

Corollary 2.2. If $c>c_{0}$ then, given $x$ in $X$, there is a number 
$\theta(x)$ such that if $\|a\|=a(x)=1$ then $\cos (\arg (b(t(x))-\theta(x))) \geqq 2 c-1$.

Proof. Let $S=\{b(t(x)) ; b=T a,\|a\|=a(x)=1\}$. Let $\theta(x)=$ $1 / 2(\sup \{\arg (z) ; z$ in $S\}+\inf \{\arg (z) ; z$ in $S\})$. If $z_{1}$ and $z_{2}$ are in $S$ then $\left|z_{i}\right| \leqq 1, i=1,2$ and by the previous theorem $\left|z_{1}+z_{2}\right| \geqq 4 c-2$. Hence, by an elementary geometric argument, for any $z$ in $S$, $\cos (\arg (z)-\theta(x)) \geqq 2 c-1$.

THEOREM 2.3. If $c>c_{0}$ then $t$ is a homeomorphism of $X$ and $Y$.

Proof. $t$ is continuous. Since $Y$ is compact it suffices to show that if $x_{n}, n=1,2, \cdots$ converge to $x_{0}$ and $y_{n}=t\left(x_{n}\right)$ converge to $y^{\prime}$, then $y^{\prime}=y_{0}=t\left(x_{0}\right)$. For $n=0,1, \cdots$ let $a_{n, 1}, a_{n, 2}, \cdots$ be a fundamental sequence at $x_{n}$. Choose $b^{\prime}$ of norm one peaking only at $y^{\prime}$. Choose $\varepsilon$ positive. By the previous theorem, for $n$ sufficiently large and for all $k, \sup _{\theta}\left\|b_{n, k}+e^{i \theta} b^{\prime}\right\| \geqq 2 c-\varepsilon$. Hence, for the same $n$ and $k$ $\sup _{\theta}\left\|a_{n, k}+e^{i \theta} a^{\prime}\right\| \geqq 2 c-\varepsilon$. Fixing $n$ sufficiently large and letting $k$ become infinite we conclude $\left|a^{\prime}\left(x_{n}\right)\right| \geqq 2 c-1-\varepsilon$. Taking the limit as $n$ becomes infinite and noting that $\varepsilon$ was arbitrary we find $\left|a^{\prime}\left(x_{0}\right)\right| \geqq$ $2 c-1$. Pick $a$ of norm one, peaking only at $x_{0}$ and with arg $a\left(x_{0}\right)=$ $\arg a^{\prime}\left(x_{0}\right)$. So, $\left\|a+a^{\prime}\right\| \geqq 2 c$. Hence $\left\|b+b^{\prime}\right\| \geqq 2 c^{2}$. Since $b^{\prime}$ could be any element of a fundamental sequence at $y^{\prime}$, this implies $\left|b\left(y^{\prime}\right)\right| \geqq$ $2 c^{2}-1 \geqq 4 c-3$. Thus, for any $a$ peaking only at $x_{0}, y^{\prime}$ is in $P_{T(a)}=$ $\{y \in Y ;|T a(y)| \geqq 4 c-3\} . \quad y_{0}$ is the intersection of all such $P_{T(a)}$. Thus $y^{\prime}=y_{0}$. This argument also holds, mutatis mutandis, for convergence over nets.

$t$ is one-to-one. Suppose $t\left(x^{\prime}\right)=t\left(x^{\prime \prime}\right)$. Let $a_{1}^{\prime}, a_{2}^{\prime}, \cdots$ be a fundamental sequence at $x^{\prime}$ and let $a_{1}^{\prime \prime}, \alpha_{2}^{\prime \prime}, \ldots$ be a fundamental sequence $a x^{\prime \prime}$. Estimating sup $\left\|a_{n}^{\prime}+e^{i \theta} a_{n}^{\prime \prime}\right\|$ and $\sup \left\|b_{n}^{\prime}+e^{i \theta} b_{n}^{\prime \prime}\right\|$ for large $n$ shows $x^{\prime}=x^{\prime \prime}$.

$t$ is onto. If not, since $t(X)$ is closed, we can find $U$ a nonempty open set in $Y$ disjoint from $t(X)$. Pick $y$ in $U, b$ in $B$ of norm one peaking only at $y$ and $|b|<2 c-1$ off $U$. Let $x$ be a point of $X$ at which $a$ peaks. By Theorem $2.1|b(t(x))| \geqq(2 c-1)\|b\|=2 c-1$. Thus $t(x)$ is in $U$ : a contradiction.

CoRollary. If there is a $T$ in $L(A, B)$ with $c(T) \geqq c_{0}$ then $X$ and $Y$ are homeomorphic.

Using the homeomorphism $t$ to identify $X$ and $Y$, the function $\theta(\cdot)$ of Corollary 2.2 can be regarded as a function on $X$ or on $Y$. For $x$ in $X$, let $\theta(x)$ be the number produced in the proof of this corollary. For $y$ in $Y$, set $\theta(y)=\theta\left(t^{-1}(x)\right)$. 
THEOREM 2.4. If $c>c_{0}$ then for all $a$ in $A$, for all $x$ in $X$, $\|b(t(x))|-| a(x)\| \leqq 2(1-c)\|a\|$.

Proof. It suffices to consider the case $\|a\|=1, a(x)$ real and positive. Let $y=t(x)$. Pick $a_{1}, a_{2}, \cdots$ a fundamental sequence for $x$ with $a_{i}(x)=1$ for all $i$. Set $r=1-a(x)$. Pick $\varepsilon$ positive. Let $x_{n}$ be the point at which $a+(1+\varepsilon) r a_{n}$ peaks. Let $y_{n}=t\left(x_{n}\right)$. By Theorem $2.1\left|b\left(y_{n}\right)+(1+\varepsilon) r b_{n}\left(y_{n}\right)\right| \geqq(2 c-1)\left\|a+(1+\varepsilon) r a_{n}\right\|>$ $2 c-1$. Hence $\left|b\left(y_{n}\right)\right| \geqq 2 c-1-(1+\varepsilon) r=2 c-2+|a(x)|-\varepsilon(1-$ $a(x))$. Letting $n$ go to infinity, $x_{n}$ approaches $x$ and hence $y_{n}$ approaches $y$ so $|b(y)| \geqq 2 c-2+|a(x)|-2 \varepsilon$. Since $\varepsilon$ was arbitrary

$$
|b(y)| \geqq 2 c-2+|a(x)| \text {. }
$$

Also,

$$
\begin{aligned}
& 1+|a(x)|=\lim _{n \rightarrow \infty} \sup _{\theta}\left\|a+e^{i \theta} a_{n}\right\| \\
& \geqq \varlimsup_{n \rightarrow \infty} \sup _{\theta}\left\|b+e^{i \theta} b_{n}\right\| \geqq \varlimsup \\
&
\end{aligned}
$$

So

$$
1+|a(x)| \geqq|b(y)|+2 c-1 .
$$

The last inequality by Theorem 2.1. Inequalities (2.2) and (2.3) imply the desired conclusion.

Theorem 2.5. Let $c>c_{0}$. Given $K, 0<K \leqq 1$, and $\varepsilon>0$, there is a $d<1$ which depends only on $K$ and $\varepsilon$ such that if $c>d$, then given $a$ of norm one and given $x$ in $X$ with $|a(x)| \geqq K$ and $\arg (a(x))=0$, then $|\arg (b(t(x))-\theta(x))|<\varepsilon$.

Proof. We may assume $a(x)$ positive. Let $y=t(x)$. Choose $a^{\prime} \neq a$ peaking only at $x$ with $a^{\prime}(x)=1$. Set $a^{\prime \prime}=\left(a-a(x) a^{\prime}\right) /\left(\left\|\alpha-a(x) a^{\prime}\right\|\right)$. $a^{\prime \prime}$ is a function of unit norm and $a^{\prime \prime}(x)=0$. Applying the previous theorem to $a^{\prime \prime}$ at the point $x$ we find $\left|b^{\prime \prime}(y)\right| \leqq 2(1-c)$. So $\mid b(y)-$ $a(x) b^{\prime}(y) \mid \leqq 2(1-c)\left\|a-a(x) a^{\prime}\right\| \leqq 4(1-c)$. So $\left|b(y) / a(x)-b^{\prime}(y)\right| \leqq$ $4(1-c) / a(x) \leqq 4(1-c) / K=\varepsilon_{1}$. Also, by applying Theorem 2.1 and Corollary 2.2 to $a^{\prime}$ at $x$ we find $\left|b^{\prime}(y)-\exp (i \theta(y))\right| \leqq \varepsilon_{2}$ where $\varepsilon_{1}$ and $\varepsilon_{2}$ depend only on $c$ and $K$ and can be chosen to be arbitrarily small if $c$ is close enough to one. Combining the last two inequalities we find $|b(y) / a(x)-\exp (i \theta(y))|<\varepsilon_{3}$ where $\varepsilon_{3}$ can be made arbitrarily small if $c$ is close enough to one. Since $|a(x)|<1$ this implies $\mid b(y)-$ $\exp (i \theta(y)) a(x) \mid<\varepsilon_{3}$. But $a(x)$ is a positive real number greater than $K$. By elementary geometry the previous inequality implies that the quantity $|\arg (b(y))-\theta(x)|$ can be made arbitrarily small by requiring 
that $\varepsilon_{3}$ be sufficiently small. This is accomplished by requiring that $c$ be larger than some constant $d$; and the theorem is proved.

2.6. Proof of Theorem A. If suffices to prove the theorem for $T$ of norm one. Let $g(y)=\exp (i \theta(y))$ and $h(y)=t^{-1}(y)$. Theorem 2.3 shows that $h$ is a homeomorphism. The previous two theorems combine to show that $g$ and $h$ satisfy the requisite inequality.

2.7. If the allowable subspaces $A$ and $B$ are also algebras and if $T(1)$ is a real positive constant, then, for $c(T)$ large enough, $T$ is almost an algebra isomorphism.

THEOREM A'. There is a function $\varepsilon(x)$ which decreases continuously to zero as $x$ increases to one and a constant $d<1$ so that if $A$ and $B$ are algebras and $T(1)$ is a positive constant and $c(T)>d$ then there is an isometric algebra isomorphism $R$ of $C(X)$ onto $C(Y)$ so that, as linear maps on $A,\|T-R\| \leqq \varepsilon(c(T))$.

Proof. Define $R f(y)=f\left(t^{-1}(y)\right)$. The theorem follows from the following lemma.

Lemma. If 1 is in $A$ then given $\varepsilon$ positive there is a $d<1$ which depends only on $\varepsilon$ so that $c>d$ implies that for all $a$ in $A$, for all $x$ in $X$

$$
|b(t(x))-\exp (i \arg (T 1)(t(x))) a(x)| \leqq \varepsilon\|a\| .
$$

Proof. We may assume $\|a\|=1$. Note that Theorem 2.1 implies that $T 1$ is bounded away from zero and hence has a well defined argument. Choose $d$ large enough so that the desired result follows immediately from Theorem 2.4 whenever $|a(x)|<\varepsilon / 3$. Now, by increasing $d$ and applying Theorem 2.5 with $K=\varepsilon / 3$ and Theorem 2.4 we can insure $|b(t(x))-\exp (i \theta(x)) a(x)|<\varepsilon / 3$. Hence it suffices to show that $|\theta(x)-\arg (T(1)(t(x)))|$ can be made small for all $x$. This, however, follows from Corollary 2.2.

COROLLARY 2.7.1. Given $\varepsilon>0$ there is a $d<1$ so that if $c>d$, $A$ and $B$ are algebras, and $T(1)$ is a positive constant, then for all $a, a^{\prime}$ in $A,\left\|(T a)\left(T a^{\prime}\right)-T\left(a a^{\prime}\right)\right\| \leqq \varepsilon\|a\|\left\|a^{\prime}\right\|$.

Proof. Let $R$ be given by the previous theorem. $T=R+$ $(T-R) . \quad R$ is multiplicative and $(T-R)$ is small. The result follows from a direct estimate using these facts.

Corollary 2.7.2. Given $K>0$, there is a $d<1$ so that if $c>$ 
$d, A$ and $B$ are algebras, and $T(1)$ is a positive constant, then, if a is invertible in $A$ and $\|a\|\left\|a^{-1}\right\| \leqq K$, then $b$ is invertible in $B$.

Proof. We may assume $a$ is of norm 1. By applying the previous corollary to the functions $a$ and $a^{-1}$ with $\varepsilon=1 /(2 K)$ we find $\left\|(T a)\left(T a^{-1}\right)-T 1\right\| \leqq 1 / 2$. But $T 1$ is a positive constant greater than $c$. By requiring that $c$ be greater than one-half, we conclude $\left\|(T a)\left(T a^{-1}\right)-1\right\|<1$. By a standard Banach algebra result we conclude that $(T a)\left(T a^{-1}\right)$, and hence also $T a$, are invertible.

2.8. The following theorem allows us to use the results of 2.7 to study the implications of $D(A, B)=0$ for function algebras.

THeOREm. If $A$ and $B$ are algebras and $D(A, B)=0$, then there is a sequence $T_{1}, T_{2}, \cdots$ of elements in $L(A, B)$ such that $T_{n}(1)=1$ for all $n$ and $\lim c\left(T_{n}\right)=1$.

Proof. Since $D(A, B)=0$ there is a sequence $S_{1}, S_{2}, \cdots$ in $L(A, B)$ with $\lim c\left(S_{n}\right)=1$. We can assume that $\left\|S_{n}\right\|=1$ for all $n$. By removing a finite number of terms from the sequence we can assume that for all $n, c\left(S_{n}\right)$ is so large that all of the previous results hold. Let $a_{n}=S_{n}^{-1}(1)$. Applying the lemma of Theorem $\mathrm{A}^{\prime}$ three times to the map $S_{n}$ and the functions $1, a_{n}$, and $a_{n}^{2}$ produces three inequalities which combine to show $\left\|S_{n}(1) S_{n}\left(a_{n}^{2}\right)-\left(S_{n}\left(a_{n}\right)\right)^{2}\right\| \leqq \varepsilon^{\prime}$ where $\varepsilon^{\prime}$ can be made arbitrarily small if the $\varepsilon$ of the lemma sufficiently small. Hence we conclude that $\left\|S_{n}(1) S_{n}\left(a_{n}^{2}\right)-1\right\|<1$. Hence $S_{n}(1)$ is invertible in $B$. Define $T_{n}$ by $T_{n}(\alpha)=\left(S_{n}(1)\right)^{-1} S_{n}(\alpha)$. It is clear that $T_{n}$ is in $L(A, B)$ and that $T_{n}(1)=1$. Theorem 2.1 allows us to conclude that $\left\|T_{n}\right\| \leqq 1 /\left(2 c\left(S_{n}\right)-1\right)$. It is a direct estimate that $\left\|T_{n}^{-1}\right\| \leqq 1 / c\left(S_{n}\right)$. The desired conclusion follows.

3. Moduli. We will use a specific set of moduli for surfaces in $\mathscr{S}$. For any $S$ in $\mathscr{S}$ we will say that $S$ is in standard position if it is realized as a subset of the complex plane bounded by $n$ circles, $C_{1}, C_{2}, \cdots, C_{n}$ with $C_{1}$ the unit circle, $C_{2}$ concentric with and outside of the unit circle, and $C_{3}$ (if there is one) with center on the $x$ axis. Let $C_{i}$ have center $x_{i}+i y_{i}$ and radius $r_{i}$.

For $S$ in standard position, we define the modulus of $S, m(S)$, to be the vector $\left(x_{3}, \cdots, x_{n}, y_{4}, \cdots, y_{n}, r_{2}, \cdots, r_{n}\right)$. When necessary, we denote the dependence of these quantities on $S$ by writing $x_{3}(S)$, etc. For $S$ in $\mathscr{S}_{n}$ with $n$ greater than $2, m(S)$ is a $(3 n-6)$ - tuple.

It is known that, given $S$ in $\mathscr{S}$, there is at least one and at most finitely many Riemann surfaces $S^{\prime}$ which are conformally equivalent to $S$ and are in standard position. (E.g. [9] 424ff.) 
We define the $m$-topology (moduli topology) on $\mathscr{S}$ as follows. A sequence $S_{1}, S_{2}, \ldots$ converges to $S$ in the $m$-topology if and only if there is a sequence $S_{1}^{\prime}, S_{2}^{\prime}, \ldots$ such that $S_{i}$ is conformally equivalent to $S_{i}^{\prime}$ for each $i$ and the vectors $m\left(S_{i}^{\prime}\right)$ approach $m(S)$ in the Euclidean topology as $i$ becomes infinite.

4. Conformal invariants and almost isometries. In this section we introduce a set of conformal invariants for the Riemann surfaces in $\mathscr{S}$, develop some of the elementary properties of these invariants and relate these invariants to $d(\cdot, \cdot)$.

Definitions 4.1. For a function algebra $A$ we define $e^{A}=$ $\exp (A)=\left\{g\right.$ in $A ; g=e^{h}$ for some $h$ in $\left.A\right\}, A^{-1}=\left\{g\right.$ in $A ; g^{-1}$ is in $\left.A\right\}$. $e^{A}$ and $A^{-1}$ are commutative groups with respect to multiplication and $e^{A}$ is a subgroup of $A^{-1}$. For $a$ in $A^{-1}$, we will denote by $(a)$ the element of the quotient group $A^{-1} / \exp (A)$ which contains $a$. For any $a$ in $A^{-1}$ we define $\rho((a))=\inf \left\{\|g\|\left\|g^{-1}\right\| ; g\right.$ in $\left.(a)\right\}$. Of course, we will often write $\rho(a)$ for $\rho((a))$.

For $n$ larger than one, let $S$ be a surface in $\mathscr{S}_{n}$. Pick a numbering of the boundary contours of $S$. For $i$ and $j$ between one and $n, i \neq j$, let $\tau_{i j}$ be the element of $A(S)$ which maps $S$ conformally onto the surface $\tau_{i j}(S)$, a surface in standard position, so that the $i$ th boundary contour of $S$ is mapped to the unit circle and the $j$ th to the circle concentric with the unit circle. The function $\rho(\cdot)$ is defined on $A(S)$. Set $\rho_{i j}(S)=\rho_{i j}=\rho\left(\left(\tau_{i j}\right)\right)$. The numbers $\rho_{i j}$ are the conformal invariants we shall consider. Note that $\rho_{i j}=\rho_{j i}$, thus for $S$ in $\mathscr{S}_{n}$ we have (at most) $n(n-1) / 2$ distinct $\rho_{i j}$. If $S$ and $S^{\prime}$ are conformally equivalent, then after some renumbering of the boundary contours of $S^{\prime}, \rho_{i j}(S)=\rho_{i j}\left(S^{\prime}\right)$ for all $i$ and $j$. (Results relating to the converse of this observation are presented in [8].)

Given $S$ in $\mathscr{S}$ and $C$ a boundary contour of $S$ and $f$ in $A(S)$ we denote by $w(C, f)$ the winding number of the curve $f(C)$ about the origin. That is, $w(C, f)$ is the winding number of $f$ on $C$.

4.2. Elementary properties. Let $H$ be the free commutative group generated by the symbols $c_{1}, c_{2}, \cdots, c_{n}$. Let $G$ be the subgroup of $H$ which consists of those elements $\sum_{i=1}^{n} a_{i} c_{i}$ for which $\sum a_{i}=0$. Let $S$ be an element of $\mathscr{S}_{n}$ with boundary contours $C_{1}, C_{2}, \cdots, C_{n}$.

THEOREM. The map $k$ from $A(S)^{-1} / \exp (A(S))$ to $G$ defined by $k((f))=\sum_{i=1}^{n} w\left(C_{i}, f\right) c_{i}$ is a group isomorphism.

Note. If $A$ is a function algebra then $A^{-1} / \exp (A) \approx H^{1}(M(A), Z)=$ the first Čech cohomology group of $M(A)$ with integer coefficients 
([6]). This theorem is just an explicit form of the isomorphism for the case of interest.

Proof. It is clear that $k$ is well defined. $k\left(\left(\tau_{i j}\right)\right)=c_{j}-c_{i}$ and elements of the form $c_{j}-c_{i}$ generate $G$, hence $k$ is onto. It remains to show that $k((f))=0$ implies that $f$ is in $\exp (A(S))$, or equivalently, $\log (f)$ is in $A(S) . \quad f$ is invertible, hence by the monodromy theorem it suffices to show that $\operatorname{im}(\log (f))$ is single valued. Any smooth curve $C$ in $S$ is homologous to $\sum_{i=1}^{n} n_{i} C_{i}$ for some choice of $n_{i}$. By direct computation the change in $1 / 2 \pi \operatorname{im}(\log (f))$ on traversing $C$ is $\sum n_{i} w\left(C_{i}, f\right)$. Since $w\left(C_{i}, f\right)=0$ for all $i$, this quantity is zero and the proof is complete.

4.3. The following reinterpretation of $\rho(\cdot)$ helps elucidate the elementary properties of $\rho(\cdot)$ and the relation between the invariants $\rho_{i j}$ and the conformal structure of $S$. Let $C_{R}(\partial S)$ be the Banach space of real valued continuous functions on the boundary of $S$. Let $\operatorname{Re} A$ be the subspace of $C_{R}(\partial S)$ consisting of real parts of functions in $A$, and let $\overline{\operatorname{Re} A}$ be the closure in $C_{R}(\partial S)$ of $\operatorname{Re} A$. Let $D$ be the Banach space $C_{R}(\partial S) / \overline{\operatorname{Re} A}$ and denote by $\|h\|_{D}$ the norm in $D$ of the coset of the element $h$ in $C_{R}(\partial S)$.

Proposition. For $f$ in $A(S)^{-1}, \log (\rho(f))=2\|\log |f|\|_{D}$.

Proof. The mapping which sends $f$ to $\log |f|$ sends $A(S)^{-1}$ into $C_{R}(\partial S)$ and $\exp A(S)$ into $\operatorname{Re} A$. The equality follows directly from the definitions.

By Theorem 4.2 the mapping $\Phi$ of $A(S)^{-1} / \exp A(S)$ to $L$, the set of points in $R^{n-1}$ with integer coordinates defined by $\Phi((f))=$ $\left(w\left(C_{1}, f\right), \cdots, w\left(C_{n-1}, f\right)\right)$ is a group isomorphism. Let $\tilde{\rho}$ be the function $\rho$ regarded via this isomorphism as a function on $L$, i.e., $\tilde{\rho}(\Phi((f)))=\log \rho((f))$.

Proposition. $\tilde{\rho}$ is the restriction to $L$ of a norm on $R^{n-1}$.

Proof. The mapping of $L$ into $D$ which sends $l$ to $\log \left|\Phi^{-1}(l)\right|$ extends to a linear map $R$ of $R^{n-1}$ into $D$. Define $\tilde{\rho}$ on $R^{n-1}$ by $\tilde{\rho}(l)=2\|R(l)\|_{D}$. The proposition will be established if we show that $R$ has kernel zero. To do this it suffices to show that $h=\sum_{i=1}^{n-1} a_{i}$ $\log \left|r_{n, i}\right|$ in $\overline{\operatorname{Re} A}$ implies $a_{i}=0, i=1, \cdots, n-1$. The period of ${ }^{*} h$, the harmonic conjugate of $h$ about $\rho_{i}$, a curve interior to $S$ and homotopic to $C_{i}$, is $2 \pi \alpha_{i}$. However $h$ is in $\overline{\operatorname{Re} A}$. Hence by a standard approximation argument this period must be zero. Thus all the $a_{i}$ are zero. The proposition is proved.

4.4. Continuity of the $\rho_{i j}$. We now show that the $\rho_{i j}$ are con- 
tinuous functions on $\mathscr{S}_{n}$ with respect to the topology induced by the metric $d(\cdot, \cdot)$.

Theorem. Given $S$ in $\mathscr{S}_{n}$ and $\varepsilon>0$ there is a $\delta$ so that if $S^{\prime}$ is in $\mathscr{S}_{n}$ and $d\left(S, S^{\prime}\right)<\delta$ then there is a renumbering of the boundary contours of $S^{\prime}$ after which $\left|\rho_{i j}(S)-\rho_{i j}\left(S^{\prime}\right)\right| \leqq \varepsilon, 1 \leqq i, j \leqq n$.

Proof. An argument similar to that in the proof of Theorem 2.8 shows that for $\delta$ small enough, $d\left(S, S^{\prime}\right)<\delta$ implies that there is a $T$ in $L\left(A(S), A\left(S^{\prime}\right)\right)$ with $c(T)>1-\delta^{\prime}$, and $T(1)=1$, where $\delta^{\prime}$ depends on $\delta$ and can be made arbitrarily small if $\delta$ is made sufficiently small. By choosing $\delta$ to be perhaps smaller still we can also insure that such a $T$ determines a homeomorphism of $\partial S$ and $\partial S^{\prime}$ in the manner described in Theorems 2.1 and 2.2. Given $S^{\prime}$ with $d\left(S, S^{\prime}\right)$ less than this new $\delta$, renumber the boundary components so as to be compatible with this homeomorphism. Denote the boundary contours of $S$ and $S^{\prime}$ by $C_{1}, \cdots, C_{n}$ and $C_{1}^{\prime}, \cdots, C_{n}^{\prime}$ respectively.

Let $K=3 \max \left\{\rho_{i j}(S) ; 1 \leqq i, j \leqq n\right\}$. Since winding numbers are integers, by choosing $\delta$ to be perhaps smaller still, Theorem 2.5 allows us to conclude that if $f$ is in $A(S)^{-1}$ with $\|f\|\left\|f^{-1}\right\|<K$ then $W\left(C_{i}, f\right)=W\left(C_{i}^{\prime}, T f\right)$ for $i=1, \cdots, n$.

Given $i$ and $j$ between 1 and $n$, choose $f$ in the coset of $A(S)^{-1} / \exp (A(S))$ which determines $\rho_{i j}$ so that $\|f\| \leqq\|T\|^{-1}$ and $\left\|f^{-1}\right\| \leqq \rho_{i j}+\varepsilon^{\prime}<K$ for some small preassigned positive $\varepsilon^{\prime}$. The remarks of the previous paragraph imply that $T f$ is in the coset of $A\left(S^{\prime}\right)^{-1} / \exp \left(A\left(S^{\prime}\right)\right)$ which determines $\rho_{i j}\left(S^{\prime}\right)$. Thus

$$
\rho_{i j}\left(S^{\prime}\right) \leqq\|T f\|\left\|(T f)^{-1}\right\| \leqq\left\|(T f)^{-1}\right\|
$$

But by Theorem 2.4

$$
\begin{aligned}
\left\|(T f)^{-1}\right\|^{-1} & =\inf \left\{|T f(z)| ; z \text { in } \partial S^{\prime}\right\} \\
& \geqq \inf \{|f(z)| ; z \text { in } \partial S\}+2(c(T)-1) \\
& \geqq\left\|f^{-1}\right\|^{-1}+2(c(T)-1) .
\end{aligned}
$$

$\varepsilon^{\prime}$ was arbitrary so $\rho_{i j}\left(S^{\prime}\right) \leqq\left(\rho_{i j}(S)^{-1}+2(c(T)-1)\right)^{-1}$. Hence $\rho_{i j}\left(S^{\prime}\right) \leqq$ $\rho_{i j}(S)+2(1-c(T)) \rho_{i j} \leqq \rho_{i j}+K(1-c(T))$. Hence, if $\delta^{\prime}$ is small enough, then the $\rho_{i j}\left(S^{\prime}\right)$ are bounded by the same $K$. In this case the role of $S$ and $S^{\prime}$ in the previous argument can be interchanged and $\rho_{i j}(S) \leqq$ $\rho_{i j}\left(S^{\prime}\right)+K(1-c(T))$. The previous two inequalities imply the desired result.

5. Construction of elements of $L\left(A(S), A\left(S^{\prime}\right)\right)$. Let $S$ be an element on $\mathscr{S}_{n}$ in standard position. The following Banach space direct sum decomposition of $A(S)$ will be called a standard decomposi- 
tion.

$$
A(S)=C \oplus A_{1} \oplus \cdots \oplus A_{n}
$$

where $A_{2}=\{f$ in $A(S), f$ is the restriction to $S$ of a function analytic interior to $C_{2}$ and vanishing at 0$\}$. For $i \neq 2, A_{i}=\{f$ in $A(S), f$ is the restriction to $S$ of a function analytic exterior to $C_{i}$ and vanishing at $\infty$.

Given $f$ in $A(S)$, set $f_{i}^{*}(w)=1 / 2 \pi i \int_{c_{i}} f(z)(z-w)^{-1} d z$. Set $f_{i}=$ $f_{i}^{*}-f_{i}^{*}\left(P_{i}\right)$ where $P_{2}=0$ and $P_{i}=\infty$ for $i \neq 2$. Since $f_{i}$ is in $A_{i}$ and $f=$ $\left(\sum f_{i}^{*}\left(P_{i}\right)\right)+f_{1}+\cdots+f_{n}$, the sum is all of $A$. Any element in $A_{i} \cap$ $A_{j}$ for $i \neq j$ would be analytic on the Riemann sphere and vanish at $\infty$ and thus would be the zero function.

For $f$ in $A(S)$, define $n(f)=\left|\sum f_{i}^{*}\left(P_{i}\right)\right|+\sum\left\|f_{i}\right\| \cdot n(\cdot)$ is a norm on $A(S)$ and by the closed graph theorem the map from $A(S)$ into $A(S)$ normed by $n(\cdot)$ is bicontinuous. The projection $P(f)=f_{i}$ is clearly continuous with respect to this new norm, hence it is continuous with respect to the original norm and the sum is a Banach space direct sum.

For $n=2$, the standard decomposition decomposes an element $f$ in $A(S)$ into the sum of the terms of the Laurant series of $F$ involving positive powers of the variable, those involving the negative powers of the variable, and the constant term.

THEOREM 5.1. If $S_{i}, i=0,1, \cdots$ are in $\mathscr{S}_{n}$ and the $S_{i}$ approach $S_{0}$ in m-topology as $i$ becomes infinite, then $\lim _{i \rightarrow \infty} d\left(S_{i}, S_{0}\right)=0$.

Proof. We will show that for any compact subset $W$ of $\mathscr{S}_{n}$ there is a continuous function $\lambda(t)$ which approaches zero as $t$ goes to zero such that if $S$ and $S^{\prime}$ are in $W$ then $d\left(S, S^{\prime}\right) \leqq \lambda\left(\left\|m(S)-m\left(S^{\prime}\right)\right\|_{\infty}\right)$.

Let $S$ and $S^{\prime}$ be any two elements of $\mathscr{S}_{n}$. We will construct a $T$ in $L\left(A(S), A\left(S^{\prime}\right)\right)$. Let $A=A(S), A^{\prime}=A\left(S^{\prime}\right)$. Without loss of generality we can assume that $S$ and $S^{\prime}$ are in standard position. Thus $\partial S$ and $\partial S^{\prime}$ are each a union of $n$ disjoint circles, $C_{1}, \cdots, C_{n}$ and $C_{1}^{\prime}, \cdots, C_{n}^{\prime}$ respectively. Let $A=A_{1} \oplus \cdots \oplus A_{n} \oplus C$ and $A^{\prime}=A_{1}^{\prime} \oplus$ $\cdots \oplus A_{n}^{\prime} \oplus C$ be the standard decompositions of $A$ and $A^{\prime}$ respectively. Let $t_{1}(z)=z$, and $t_{2}(z)=\left(r_{2}^{\prime} / r_{2}\right) z\left(r_{2}^{\prime}\right.$ and $r_{2}$ are the radii of $C_{2}^{\prime}$ and $C_{2}$ respectively). For $k=3,4, \cdots, n$, let $t_{k}$ be the Mobius transformation which takes the exterior of $C_{k}$ to the exterior of $C_{k}^{\prime}$ fixes $\infty$ and moves $C_{k}$ as little as possible, i.e., subject to the two previous conditions minimize sup $\left\{\left|z-t_{k}(z)\right|: z\right.$ in $\left.C_{k}\right\}$.

For $f$ in $A$ we have $f=c+\sum f_{i}$ with $c$ a constant and $f_{i}$ in $A_{i}$. Let $(T f)(w)=c+\sum f_{i}\left(t_{i}^{-1}(w)\right)$. Note that for each $i, T$ restricted to $A_{i}$ is a subjective isometry of $A_{i}$ onto $A_{i}^{\prime}$ and that the projection of 
$A$ onto $A_{i}$ is continuous. Hence $T$ is in $L\left(A, A^{\prime}\right)$.

For each $k$ we have $T_{k}$ mapping $A\left(S_{k}\right)$ to $A\left(S_{0}\right)$ constructed according to the prescription just given with $S=S_{k}$ and $S^{\prime}=S_{0}$. It suffices to show $\overline{\lim }_{n \rightarrow \infty} \log \left\|T_{n}\right\|\left\|T_{n}^{-1}\right\|=0$. Notice that $T_{n}^{-1}$ is the map that we would have constructed according to the above prescription had we interchanged $S$ and $S^{\prime}$ before starting. We will show that $\varlimsup \lim \left\|T_{n}\right\|=1$. Since $T_{n}(1)=1$ we know that $\left\|T_{n}\right\| \geqq 1$ for all $n$. The same estimates with the requisite changes in subscripts would also show that $\varlimsup_{\lim }\left\|T_{n}^{-1}\right\|=1$. We will not provide the details for this second claim.

We may limit consideration to those $S_{k}$ for which $m\left(S_{k}\right)$ lies in $W$, some preassigned compact neighborhood of $m\left(S_{0}\right)$. This having been done, the constants $R, K$, and $M$ of the next three lemmas may be chosen as universal constants, that is depending on $W$ but independent of the choice of $S$ in $\mathscr{S}_{n}$. (This uniformity follows from the fact that the constant $R$ in the proof of Lemma 1 can be bounded away from 1 for all $S$ with $m(S)$ in $W$.)

LEMMA 1. There is a positive number $k$ such that given $j, 1 \leqq$ $j \leqq n$, and given $c$ in $C, f_{i}$ in $A_{i}, f=c+\sum f_{i}$ with $\left\|f_{j}\right\|=1$ and $\left\|c+\sum f_{i}\right\|_{c_{j}}=\left\|c+\sum f_{i}\right\|$ (i.e., $f$ peaks on $C_{j}$ ), then $\left\|c+\sum f_{i}\right\| \geqq k$.

Proof of lemma. Assume $j \neq 2$ (the case $j=2$ requires minor notational changes). Assume $\|f\|=\|f\|_{c_{j}} \leqq k$. Draw a circle $\Gamma$, "hyperbolically concentric" with $C_{j}$, i.e., if one maps ext $\left(C_{j}\right)$ conformally to the unit disk and $\infty$ to 0 , then the image of $\Gamma$ will be concentric with the unit circle. We also require that the region between the two circles be contained in $S$. By Schwarz's lemma we have

$$
\left\|f-f_{j}\right\|_{r} \leqq\|f\|_{r}+\left\|f_{j}\right\|_{r} \leqq k+1 / R \text {. }
$$

The constant $R$ which is greater than 1 is determined by the relative positions of $\Gamma$ and $c_{j}$. Hence by the maximum modulus principle for (int $\Gamma$ ) applied to the function $f-f_{j}$ we have $\left\|f-f_{j}\right\|_{c_{j}} \leqq\left\|f-f_{j}\right\|_{\Gamma}$. So,

$$
\begin{aligned}
k & \geqq\|f\|=\|f\|_{c_{j}} \geqq\left\|f_{j}\right\|_{c_{j}}-\left\|f-f_{j}\right\|_{c_{j}} \\
& \geqq 1-\left\|f-f_{j}\right\|_{r} \geqq 1-k-1 / R .
\end{aligned}
$$

So $k \geqq(1-1 / R) / 2$. The same argument can be used for any $j$ (with the obvious modifications if $j=2$ ). Hence taking $k$ to be the minimum of the finite number of k's that are produced by such arguments, the lemma is proved.

Lemma 2. There is a $K$ such that if $f_{i}$ is in $A_{i}$ and if for some 
$j,\left\|c+\sum f_{i}\right\|=\left\|c+\sum f_{i}\right\|_{c_{j}}=1$ then $\left\|f_{j}\right\| \leqq K$

Proof of lemma. Let $f_{i}^{\prime}=f_{i} /\left\|f_{j}\right\|$ for $i$ equal one through $n$. Set $c^{\prime}=c /\left\|f_{j}\right\|$. Apply Lemma 1 to $f^{\prime}=c^{\prime}+\sum f_{i}^{\prime}$. So $1 /\left\|f_{j}\right\|=$ $\left\|f^{\prime}\right\| \geqq k$. So $\left\|f_{j}\right\| \leqq 1 / k=K$.

Lemma 3. There is an $M$ such that, given $f$ in $A,\|f\|=1$, then $\left\|f_{i}\right\| \leqq M$ for all $i$.

Proof. Let $f=c+\sum f_{j}$ with $f_{j}$ is $A_{j}$. Assume $\|f\|=\|f\|_{c_{i}}$. By the previous lemma, $\left\|f_{i}\right\| \leqq K$. Let $g=\left(f-f_{i}\right) /\left\|f-f_{i}\right\|$. For some $j \neq i,\|g\|=\|g\|_{c_{j}}$. Denote the projection of $g$ in $A_{j}$ by $g_{j}$. By the previous lemma, $\left\|g_{j}\right\| \leqq K$. Hence $\left\|f_{j}\right\|=\left\|f-f_{j}\right\|\left\|g_{j}\right\| \leqq$ $(1+K) K$. Continuing in this manner (i.e., next $\left.h=\left(g-g_{j}\right) /\left\|g-g_{j}\right\|\right)$ gives a bound on all the $f_{k}$. Taking the greatest of these bounds, $\left\|f_{j}\right\| \leqq K(1+K)^{n}$ for all $j$.

Note. This estimate (Lemma 3) also follows from the continuity of the projections of $A$ onto each of the summands $A_{i}$. However, the constant produced by that observation depends on the particular surface rather than on the compact set $W$.

LEMma 4. There is a function $M\left(i, j, m(S), m\left(S^{\prime}\right)\right)$ such that if $f$ is in $A_{i}$ and $z_{0}$ is in $c_{j}$ for $i \neq j$, then $\left|f_{i}\left(z_{0}\right)-f_{i}\left(t_{j}^{-1}\left(z_{0}\right)\right)\right| \leqq\left\|f_{i}\right\|$ $M\left(i, j, m(S), m\left(S^{\prime}\right)\right)$ and $M\left(i, j, m(S), m\left(S^{\prime}\right)\right)$ approaches zero as $\left\|m(S)-m\left(S^{\prime}\right)\right\|_{\infty}$ approaches zero with $S$ and $S^{\prime}$ restricted to lie in a preassigned compact subset $W$ of $\mathscr{S}_{n}$.

Proof of lemma. For simplicity we will assume that neither $i$ nor $j$ is equal to two. Let $D_{i}=\left(\right.$ exterior of $\left.C_{i}\right) \cup\{\infty\}$. Let $f_{i}^{\prime}=$ $f_{i} /\left\|f_{i}\right\|$. By Pick's lemma,

$$
\delta\left(f_{i}^{\prime}\left(z_{0}\right)\right), f_{i}^{\prime}\left(t_{i}^{-1}\left(z_{0}\right)\right) \leqq \delta_{D_{i}}\left(z_{0}, t_{i}^{-1}\left(z_{0}\right)\right)
$$

Also

$$
\frac{1}{2}\left|f_{i}^{\prime}\left(z_{0}\right)-f_{i}^{\prime}\left(t_{i}^{-1}\left(z_{0}\right)\right)\right| \leqq \delta\left(f_{i}^{\prime}\left(z_{0}\right)\right), f_{i}^{\prime}\left(t_{i}^{-1}\left(z_{0}\right)\right),
$$

hence

$$
\frac{1}{2}\left|f_{i}\left(z_{0}\right)-f_{i}\left(t_{i}^{-1}\left(z_{0}\right)\right)\right| \leqq\left\|f_{i}\right\| \delta_{D_{i}}\left(z, t_{i}^{-1}\left(z_{0}\right)\right)
$$

We $\quad$ set $M\left(i, j, m(S), m\left(S^{\prime}\right)\right)=2 \sup \left[\delta_{D_{i}}\left(z, t_{i}^{-1}(z)\right): z\right.$ in $\left.\left(c_{j}\right)\right]$. Since $M\left(i, j, m(S), m\left(S^{\prime}\right)\right)$ is continuous with respect to $m(S)$ and $m\left(S^{\prime}\right)$ the 
lemma is proved.

Proof of the theorem. Pick $S_{k}$ and $S_{0}$. Let $S_{k}=S, S_{0}=S^{\prime}$. Let $T$ be the map of $A$ onto $A^{\prime}$ defined above. Pick $f$ in $A$ with $\|f\|=1$. We will estimate $\|T\|$ by estimating $\|T f\|$. We will assume that $f$ peaks on $C_{i}$ and will only estimate $\|T f\|_{c^{\prime} \cdot}$ Similar estimates apply to the other $\|T f\|_{c^{\prime} \cdot}$ Pick $z_{0}^{\prime}$ in $C_{i}$. Let $f=c+\sum_{i} f_{i}$ with $c$ in $C$ and $f_{i}$ in $A_{i}$. Let $z_{0}=t_{i}^{-1}\left(z_{0}^{\prime}\right)$. Thus $z_{0}$ is in $C_{i}$. We have $f\left(z_{0}\right)=c+\sum_{i} f_{i}\left(z_{0}\right)$. So

$$
\begin{aligned}
\left|T f\left(z_{0}^{\prime}\right)-f\left(z_{0}\right)\right| & =\left|\sum f_{j}\left(z_{0}\right)-\sum f_{j}\left(t_{j}^{-1}\left(z_{0}^{\prime}\right)\right)\right| \\
& =\left|\sum_{j \neq i}\left(f_{j}\left(z_{0}\right)-f_{j}\left(t_{j}^{-1}\left(z_{0}^{\prime}\right)\right)\right)\right| \\
& \leqq \sum_{j \neq i}\left|f_{j}\left(z_{0}\right)-f_{j}\left(t_{j}^{-1}\left(z_{0}^{\prime}\right)\right)\right| \\
& \leqq \sum_{j \neq i}\left\|f_{j}\right\| M\left(i, j, m(S), m\left(S^{\prime}\right)\right) \text { by Lemma } 4 \\
& \leqq M^{\prime}\left(m(S), m\left(S^{\prime}\right)\right) \sum_{j \neq i}\left\|f_{j}\right\| \text { with } M^{\prime} \text { defined the obvious way } \\
& \leqq M^{\prime}\left(m(S), m\left(S^{\prime}\right)\right) \sum M \text { by Lemma } 3 \\
& \leqq N M^{\prime}\left(m(S), m\left(S^{\prime}\right)\right) \text { for some constant } N .
\end{aligned}
$$

So $\left|T f\left(z_{0}^{\prime}\right)\right| \leqq\left|f\left(z_{0}\right)\right|+N M^{\prime}\left(m(S), m\left(S^{\prime}\right)\right) \leqq 1+N M^{\prime}\left(m(S), m\left(S^{\prime}\right)\right)$. Once the compact set $W$ in $\mathscr{S}_{n}$ has been fixed then the constant $N$ in the previous inequality can be chosen uniformly and hence absorbed into the function $M^{\prime}(\cdot, \cdot) . \quad z_{0}$ was an arbitrary point on $\overline{\partial S^{\prime \prime}}$. Taking the supremum over all such $z_{0}$ we have

$$
\|T f\| \leqq|f|+M^{\prime}\left(m(S), m\left(S^{\prime}\right)\right) .
$$

Taking the supremum over all $f$ in $A$ of norm one we find

$$
\|T\| \leqq 1+M^{\prime}\left(m(S), m\left(S^{\prime}\right)\right) .
$$

Since $M^{\prime}$ has the required properties, the proof is complete.

6. Almost isometries and moduli of domains. In this section we prove the results necessary to complete the proof of Theorem B. The major remaining steps are the following two theorems.

THEOREM 6.1. Given $n \geqq 2, S$ and $S^{\prime}$ in $\mathscr{S}_{n}$, if $d\left(S, S^{\prime}\right)=0$ then $S$ and $S^{\prime}$ are conformally equivalent.

Proof. Set $A=A(S), A^{\prime}=A\left(S^{\prime}\right)$. By Theorem 2.8 there is a sequence of maps $T_{i}$ in $L\left(A, A^{\prime}\right)$ such that $c\left(T_{i}\right)$ approaches one and $T_{i}(1)=1$. Without changing notation we normalize these $T_{i}$ so that they are all of norm one. Hence, $T_{i}(1)$ will be a positive constant 
between $c\left(T_{i}\right)$ and one. By Theorem 2.2, for sufficiently large $i$, each $T_{i}$ has associated with it a homeomorphism of the boundary of $S$ with that of $S^{\prime}$. This homeomorphism induces a renumbering of the boundary components of $S^{\prime}$. Since there are only finitely many possible renumbering of the boundary components of $S^{\prime}$, we may pass to an infinite subsequence of the $T_{i}$ 's with the $c\left(T_{i}\right)$ 's large enough to insure that the conclusions of Theorem 2.2 hold and with all of the associated boundary homeomorphisms inducing the same renumbering of the boundary components of $S^{\prime}$. We will denote this new sequence by $\left\{T_{i}\right\}$ and will assume that the boundary contours have been renumbered so that for all $j$ the induced homeomorphism carries the $j$ th boundary component of $S$ to the $j$ th boundary component of $S^{\prime}$.

The proof now consists of constructing an analytic map from $S^{\prime}$ into $S$, showing that the induced map between homology groups has kernel zero and concluding that the map is a conformal equivalence. Most of the previous results in this paper worked with the boundary points of the surfaces being considered. In this proof we will only show that int $(S)$ and int $\left(S^{\prime}\right)$ are conformally equivalent. The equivalence of the two as bordered surfaces then follows from standard results about the boundary behavior of conformal maps.

For convenience we will break the proof into a series of lemmas.

LEMma 1. There is a subsequence $\left\{T_{n_{i}}\right\}$ of the $T_{i}$ 's such that for each $y$ in the interior of $S^{\prime}$ there is a point $x(y)$ in $S$ such that for all $f$ in $A \lim _{i \rightarrow \infty}\left(T_{n_{i}} f\right)(y)=f(x(y))$. Furthermore, the mapping that sends $y$ to $x(y)$ is analytic.

Proof of lemma. Pick $y$ in $S^{\prime}$. All of the $T_{n}$ are of norm one, hence all of the $T_{n}^{*}$ are of norm one. Hence, by the weak-star compactness of the unit ball of $A^{*}$, the set $\left\{T_{n}^{*}(y)\right\}$ has a weak-star accumulation point $x(y)$.

We now perform two diagonalizations on the sequence $\left\{T_{i}\right\}$. Let $f_{1}, f_{2}, \cdots$ be a countable dense subset of $A$. (Since $A$ is a direct sum of disk algebras with their constants identified, this is clearly possible.) We know that $\left\{T_{n}^{*}(y)\left(f_{1}\right)\right\}$ has the point $x(y)\left(f_{1}\right)$ as an accumulation point. By passing to a subsequence of the $T_{i}$ 's and renumbering we can insure that

$$
\lim _{n \rightarrow \infty} T_{n}^{*}(y)(f)=x(y)(f)
$$

for this particular $y$ and for $f=f_{1}$. By passing to a further subsequence we can insure that (6.1) holds for this $y$ and for $f_{2}$. Continuing in this manner and then replacing $\left\{T_{i}\right\}$ by the diagonal subsequence we insure that (6.1) holds for this $y$ and for all $f_{i}$. Since the 
$f_{i}$ are dense we can conclude that (6.1) holds for this $y$ and for all $f$ in $A$.

Let $y_{1}, y_{2}, \ldots$ be a countable dense subset of the interior of $S^{\prime}$. By another diagonalization, this time with respect to the $y_{i}$ 's, we obtain a subsequence of the $T_{i}$ 's such that (6.1) holds for all $f$ in $A$ and for $y$ equal to any of the $y_{i}$. For the other $y$ in $S^{\prime}$ we let $x(y)$ be any weak-star accumulation point of the sequence $\left\{T_{n}^{*}(y)\right\}$. In fact (6.1) now holds for all $f$ in $A$ and for all $y$ in the interior of $S^{\prime}$; for, by construction, $x(y)(f)$ is an accumulation point of the numbers $T_{n}^{*}(y)(f)$. Hence it suffices to show that this sequence has only one accumulation point. $y$ is an interior point of $S^{\prime}$ and, for fixed $f$, the $T_{n}(f)$ are uniformly bounded on $S^{\prime}$. Hence, in a small neighborhood of $y$, this set of functions is uniformly equicontinuous. But for a dense set of points, $y_{i}$, in this neighborhood, the sequences $\left\{T_{n}^{*}\left(y_{i}\right)(f)\right\}$ have unique accumulation points. Hence the sequence $\left\{T_{n}^{*}(y)(f)\right\}$ cannot have more than one accumulation point.

We now show that for any $y, x(y)$ is a nonzero multiplicative linear functional on $A^{\prime} . \quad x(y)(1)=\lim \left(T_{n}^{*}(y)(1)\right)=\lim T_{n}(1)(y)=1$. Hence $x(y)$ is nonzero. It remains to show that for any $f$ in $A$ of norm one $x(y)\left(f^{2}\right)=(x(y)(f))^{2}$. We know that $\lim \left(T_{n}^{*}(y)(f)\right)^{2}=$ $(x(y)(f))^{2}$ and that $\lim \left(T_{n}^{*}(y)\left(f^{2}\right)\right)=x(y)\left(f^{2}\right)$. We also know that $\left|\left(T_{n}^{*}(y)(f)\right)^{2}-T_{n}^{*}(y)\left(f^{2}\right)\right| \leqq\left\|\left(T_{n} f\right)^{2}-T_{n}\left(f^{2}\right)\right\|$ and by Corollary 2.7.1 this last quantity must become arbitrarily small as $n$ becomes infinite. Combining these observations show that $x(y)\left(f^{2}\right)=(x(y)(f))^{2}$. Since $x(y)$ is a multiplicative linear functional, it can be thought of as a point of $S$, the maximal ideal space of $A=A(S)$. We will denote this point by $x(y)$.

We now show that this mapping from $y$ to $x(y)$ is analytic. Fix $y$ in the interior of $S^{\prime}$. Let $h$ be the coordinate function on $S$. We know that the sequence of functions $\left\{T_{n} h(y)\right\}$ converges pointwise to the function $h(x(y))=x(y)$. We also know that the sequence of functions $T_{n} h$ is a bounded sequence of analytic functions on $S^{\prime}$. These two facts allow us to conclude that a subsequence of the $T_{n} h$ converge uniformly on some small neighborhood of $y$ to the limit function $x$. Thus, at $y$, the function $x(y)$ is the uniform limit of the analytic functions $T_{n} h$, and hence is analytic. $y$ was arbitrary so the lemma is proved.

Note. We have not ruled out the possibility that $x(y)$ is a constant function. Simple examples using the disk algebra show that this is, in fact, possible if we do not require $S$ and $S^{\prime}$ each have more than one boundary component.

The presence of homology in $S$ and $S^{\prime}$ prevents the map from being trivial. We have identified the integer cohomology groups 
$H^{1}(S)$ and $H^{1}\left(S^{\prime}\right)$ with $A^{-1} / e^{A}$ and $A^{\prime-1} / e^{A^{\prime}}$ respectively. Since $x$ maps $S^{\prime}$ to $S$ there is an induced map $x^{*}$ of $H^{1}(S)$ into $H^{1}\left(S^{\prime}\right)$.

\section{LEMMA 2. $\operatorname{Ker} x^{*}=0$.}

Proof. For $i=1, \cdots, n-1$ let $\gamma_{i}$ be a simple closed curve in the interior of $S$ which is homotopic to the ith boundary contour of $S$ and is similarly oriented. Define $\gamma_{i}^{\prime}$ in $S^{\prime}$ similarly. Suppose that $f$ is in $A^{-1}$ and $(f)$ in $A^{-1} / e^{A}$ is such that $x^{*}(f)=0$. We must show that $f$ is in $e^{A}$. It suffices to show that $W\left(\gamma_{i}, f\right)=0$ for each $i$. Choose $i$. Since $x^{*}(f)=0$, we know that $x^{*}(f)\left(\gamma_{i}^{\prime}\right)=W\left(\gamma_{i}^{\prime}, f \circ x\right)=$ 0 . Since $\gamma_{i}^{\prime}$ is a compact subset of the interior of $S^{\prime}, W\left(\gamma_{i}^{\prime}, f \circ x\right)=$ $\lim W\left(\gamma_{i}^{\prime}, T_{n} f\right)$. Since the winding numbers are integers this implies that for all $n$ sufficiently large $W\left(\gamma_{i}^{\prime}, T_{n} f\right)=0$. Let $C_{i}$ and $C_{i}^{\prime}$ be the $i$ th boundary contours of $S$ and $S^{\prime}$ respectively. $W\left(\gamma_{i}^{\prime}, T_{n} f\right)=$ $W\left(C_{i}^{\prime}, T_{n} f\right)$. Hence for all $n$ sufficiently large, $W\left(C_{i}^{\prime}, T_{n} f\right)=0$. Hence, by the lemma of Theorem $\mathrm{A}^{\prime} W\left(C_{i}, f\right)=0$. But $W\left(\gamma_{i}, f\right)=$ $W\left(C_{i}, f\right)$ so the proof is complete.

Proof of theorem. Let the mapping of $y$ to $x(y)$ be the map of the interior of $S^{\prime}$ into $S$ constructed in Lemma 1 . Applying Lemma 1 again in the opposite direction gives a map of the interior of $S$ into $S^{\prime}$ which sends $x$ to $y(x)$. Let $K$ be the map of the interior $S$ into itself defined by $K(x)=x(y(x))$. The mapping of $x$ into $y(x)$ is only defined on the interior of $S$. However, the previous lemmas show that the map of $y$ to $x(y)$ is nonconstant and analytic. Hence the image of the interior of $S^{\prime}$ is contained in the interior of $S$. Thus $K$ is well defined. $K$ induces a map $K^{*}$ of $H^{\prime}(S)$ into itself and $K^{*}=y^{*} x^{*}$. By Lemma 2, $\operatorname{Ker} x^{*}=0$ and $\operatorname{Ker} y^{*}=0$. Hence $\operatorname{Ker} K^{*}=0$. Since $H^{1}(S)$ and $H_{1}(S)$, the integer homology group of $S$, are both free on $n-1$ generators, we may conclude that $K_{*}$ mapping $H_{1}(S)$ to itself has trivial kernel. Landau and Osserman [4] have shown that if $S$ is a finite planar domain and $K$ is an analytic map of $S$ into itself such that $K_{*}$ has trivial kernel, then $K$ is a conformal automorphism. Thus $K$, and similarly $H=y \circ x$ mapping $S^{\prime}$ to itself, are conformal automorphisms. Hence $x$ is a conformal isomorphism of $S$ and $S^{\prime}$ and the theorem is proved.

THEOREM 6.3. If $S_{k}$ is in $\mathscr{S}_{n}, k=0,1,2, \cdots$ and $\lim d\left(S_{k}, S_{0}\right)=$ 0 then $\left\{S_{k}\right\}_{k=0}^{\infty}$ lies in an m-topology compact subset of $\mathscr{S}_{n}$.

Proof. Put $S_{0}$ in standard position. Let $S_{0}-\varepsilon$ be the set of all points in $S_{0}$ of distance at least $\varepsilon$ from $\partial S_{0}$. If $\varepsilon$ is sufficiently small then $S_{0}-\varepsilon$ and $S_{0}-2 \varepsilon$ are in $\mathscr{S}_{n}$. By Theorem 2.8, for $k$ sufficiently 
large we can find $T_{k}$ in $L\left(A\left(S_{k}\right), A\left(S_{0}\right)\right)$ with $T_{k}(1)=1$ and $\lim c\left(T_{k}\right)=1$. For $N$ large and $\varepsilon$ small, Theorem $\mathrm{A}^{\prime}$ holds and hence for all $n>N$, for all $x$ in $\partial S_{0}$

$$
\left|T_{n}\left(\tau_{12}\right)\left(t_{n}(x)\right)-\tau_{12}(x)\right|<\varepsilon / 2
$$

where $t_{n}$ is the boundary homeomorphism induced by $T_{n}$ in the manner described in Theorem 2.3. If $\alpha$ is in $S_{0}-\varepsilon$ then, by (6.2), since winding numbers are integers $1=W\left(\partial S_{0}, \tau_{12}-\alpha\right)=W\left(\partial S_{n}, T\left(\tau_{12}\right)-\alpha\right)$. Hence $T_{n}\left(\tau_{12}\right)$ map a subset of $S_{n}$ univalently onto $S_{0}-\varepsilon$. Let $h_{n}$ be the inverse of this map. Thus $h_{n}$ maps $S_{0}-\varepsilon$ into $S_{n}$ and the components of $C-S_{n}$ are contained in the components of $C-h_{n}\left(S_{0}-\varepsilon\right)$. Since $c\left(T_{n}\right)$ approach one, the functions $h_{n}$ are uniformly bounded by some constant $K$. Hence, the set of numbers $\left\{r_{2}\left(S_{k}\right)\right\}$ is bounded for, if not, then the numbers $\rho_{12}\left(S_{n}\right) \geqq r_{2}\left(S_{n}\right) / K$ would not be bounded, an impossibility by Theorem 4.4.

For $S$ in $\mathscr{S}_{n}$ and $t$ a continuous function on $S$, define $B(t, S)=$ $\inf \{|t(x)-t(y)| ; x$ and $y$ in different boundary components of $\partial S\}$. Let $B(S)$ be $B(t, S)$ with $t$ the map which puts $S$ in standard position.

Note that if some sequence of $r_{i}\left(S_{n}\right)$ approach zero as $n$ becomes infinite and if the $B\left(S_{n}\right)$ are bounded away from zero then some of the $\rho_{i j}\left(S_{n}\right)$ must become arbitrarily large. Again, by Theorem 4.4, this is impossible.

The only way in which the moduli of the $S_{n}$ can fail to lie in a compact subset are if the $r_{2}\left(S_{n}\right)$ are unbounded, or if some $r_{i}\left(S_{n}\right)$ become arbitrarily small, or if some $B\left(S_{n}\right)$ become arbitrarily small. We have already ruled out the first possibility and shown the second possibility cannot happen unless some $B\left(S_{n}\right)$ are arbitrarily small. We must now show that the $B\left(S_{n}\right)$ are bounded away from zero.

Suppose that, after passing to a subsequence, $B\left(S_{n}\right)$ approach zero. Since $B\left(h_{n}, S_{0}-\varepsilon\right) \leqq B\left(S_{n}\right)$ we would have $B\left(h_{n}, S_{0}-\varepsilon\right)$ approaching zero. Similarly, letting $k_{n}$ be $h_{n}$ restricted to $S_{0}-2 \varepsilon$ we would have $B\left(k_{n}, S_{0}-2 \varepsilon\right)$ approaching zero. The $h_{n}$ are a uniformly bounded family of univalent functions on $S_{0}-\varepsilon$; hence, after passing to a subsequence we can find $h$ the uniform limit on $S_{0}-3 \varepsilon / 2$ of the $h_{n}, h$ is constant or univalent. Since $W\left(|z|=1-2 \varepsilon ; h_{n}\right)=1$ for all $n, h$ is not constant. Let $k$ be $h$ restricted to $S_{0}-2 \varepsilon$. Since $k$ is univalent on $S_{0}-2 \varepsilon, B\left(k, S_{0}-2 \varepsilon\right)>0$. But $B\left(k, S_{0}-2 \varepsilon\right) \leqq \underline{\lim } B\left(k_{n}, S_{0}-\right.$ $2 \varepsilon)=0$. This contradiction completes the proof.

6.4. Proof of Theorem B. It suffices to show the following:

(1) $d(\cdot, \cdot)$ is a metric on $\mathscr{S}_{n}$ and

(2) Let $S_{0}, S_{1}, \cdots$ be elements on $\mathscr{S}_{n}$ then

(a) if the $S_{k}$ approaches $S_{0}$ in the $m$-topology then $d\left(S_{k}, S_{0}\right)$ approaches zero. 

$m$-topology.

(b) if $d\left(S_{k}, S_{0}\right)$ approaches zero then $S_{k}$ approaches $S_{0}$ in the

For 1 the only nontrivial fact is that $d\left(S, S^{\prime}\right)=0$ implies $S$ and $S^{\prime}$ are conformally equivalent. This is Theorem 6.1. 2(a) is Theorem 5.3. For 2(b) it suffices to show that the sequence $S_{k}$ has an $m$-topology accumulation point, then by the triangle inequality for $d$ and part 2(a), this point must be $S_{0}$. However, Theorem 6.2 guarantees that such an accumulation point exists.

Added in proof. A result very similar to Theorem 2.3 has been proved by B. Cengiz (Proc. Amer. Math. Soc., 40 (1973), 426-430).

\section{REFERENCES}

1. S. Banach, Theorie des Operations Lineaires, Warsaw, 1932.

2. M. Cambern, A generalized Banach-Stone theorem, Proc. Amer. Math. Soc., 17 (1966), 396-400.

3. - On isomorphisms with small bounds, Proc. Amer. Math. Soc., 18 (1967), $1062-1066$.

4. H. J. Landau and R. Osserman, On analytic mappings of Riemann surfaces, J. d'Analyse Mathematique, 7 (1959-60), 249-279.

5. M. Nakai, Algebraic criterion on quasiconformal equivalance of Riemann surfaces, Nagoya Math. J., 16 (1960), 157-184.

6. H. L. Royden, Function algebras, Bull. Amer. Math. Soc., 69 (1963), 281-298.

7. R. Rochberg, Almost isometries of Banach spaces and moduli of Riemann surfaces, Duke Math. J., 40 (1973), 41-52.

8. - Function algebra invariants and the conformal structure of planar domains, J. Functional Analysis, 13 (1973), 154-172.

9. M. Tsuji, Potential Theory in Modern Function Theory, Maruzen Co. Ltd., Tokyo, 1959 .

Received Junuary 31, 1972 and in revised form February 25, 1973. Most of the results in this paper are from the author's doctoral dissertation written at Harvard University under the direction of Professor A. Gleason. The author was partially supported by a National Science Foundation graduate fellowship.

WASHINGTON UNIVERSITY 


\section{PACIFIC JOURNAL OF MATHEMATICS}

\section{EDITORS}

RICHARD ARENS (Managing Editor)

University of California

Los Angeles, California 90024

\section{R. A. Beaumont}

University of Washington

Seattle, Washington 98105

\section{J. DuGundJI*}

Department of Mathematics

University of Southern California

Los Angeles, California 90007

D. Gilbarg and J. Milgram

Stanford University

Stanford, California 94305

ASSOCIATE EDITORS
E. F. BECKENBACH
B. H. NeumanN
F. WOLF
K. YoSHIDA

\section{SUPPORTING INSTITUTIONS}

UNIVERSITY OF BRITISH COLUMBIA

CALIFORNIA INSTITUTE OF TECHNOLOGY

UNIVERSITY OF CALIFORNIA

MONTANA STATE UNIVERSITY

UNIVERSITY OF NEVADA

NEW MEXICO STATE UNIVERSITY

OREGON STATE UNIVERSITY

UNIVERSITY OF OREGON

OSAKA UNIVERSITY

\author{
UNIVERSITY OF SOUTHERN CALIFORNIA \\ STANFORD UNIVERSITY \\ UNIVERSITY OF TOKYO \\ UNIVERSITY OF UTAH \\ WASHINGTON STATE UNIVERSITY \\ UNIVERSITY OF WASHINGTON \\ AMERICAN MATHEMATICAL SOCIETY \\ NAVAL WEAPONS CENTER
}

The Supporting Institutions listed above contribute to the cost of publication of this Journal, but they are not owners or publishers and have no responsibility for its content or policies.

Mathematical papers intended for publication in the Pacific Journal of Mathematics should be in typed form or offset-reproduced, (not dittoed), double spaced with large margins. Underline Greek letters in red, German in green, and script in blue. The first paragraph or two must be capable of being used separately as a synopsis of the entire paper. Items of the bibliography should not be cited there unless absolutely necessary, in which case they must be identified by author and Journal, rather than by item number. Manuscripts, in duplicate if possible, may be sent to any one of the four editors. Please classify according to the scheme of Math. Rev. Index to Vol. 39. All other communications to the editors should be addressed to the managing editor, or Elaine Barth, University of California, Los Angeles, California, 90024.

50 reprints are provided free for each article; additional copies may be obtained at cost in multiples of 50 .

The Pacific Journal of Mathematics is issued monthly as of January 1966. Regular subscription rate: $\$ 48.00$ a year (6 Vols., 12 issues). Special rate: $\$ 24.00$ a year to individual members of supporting institutions.

Subscriptions, orders for back numbers, and changes of address should be sent to Pacific Journal of Mathematics, 103 Highland Boulevard, Berkeley, California, 94708.

\section{PUBLISHED BY PACIFIC JOURNAL OF MATHEMATICS, A NON-PROFIT CORPORATION}

Printed at Kokusai Bunken Insatsusha (International Academic Printing Co., Ltd.), 270, 3-chome Totsuka-cho, Shinjuku-ku, Tokyo 160, Japan.

* C. R. DePrima California Institute of Technology, Pasadena, CA 91109, will replace J. Dugundji until August 1974.

Copyright (C) 1973 by

Pacific Journal of Mathematics

All Rights Reserved 


\section{Pacific Journal of Mathematics}

\section{Vol. 49, No. $2 \quad$ June, 1973}

Wm. R. Allaway, On finding the distribution function for an orthogonal polynomial

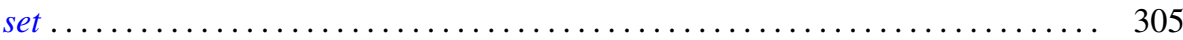

Eric Amar, Sur un théorème de Mooney relatif aux fonctions analytiques bornées... . 311

Robert Morgan Brooks, Analytic structure in the spectrum of a natural system . . . . 315

Bahattin Cengiz, On extremely regular function spaces . . . . . . . . . . . . . . 335

Kwang-nan Chow and Moses Glasner, Atoms on the Royden boundary . . . . . . . . . 339

Paul Frazier Duvall, Jr. and Jim Maxwell, Tame $Z^{2}$-actions on $E^{n} \ldots \ldots \ldots \ldots \ldots . .349$

Allen Roy Freedman, On the additivity theorem for $n$-dimensional asymptotic

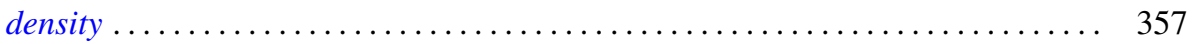

John Griffin and Kelly Denis McKennon, Multipliers and the group $L_{p}$-algebras . . . 365

Charles Lemuel Hagopian, Characterizations of $\lambda$ connected plane continua ....... 371

Jon Craig Helton, Bounds for products of interval functions . . . . . . . . . . . 377

Ikuko Kayashima, On relations between Nörlund and Riesz means . . . . . . . . . . 391

Everett Lee Lady, Slender rings and modules . . . . . . . . . . . . . . . . . . 397

Shozo Matsuura, On the Lu Qi-Keng conjecture and the Bergman representative

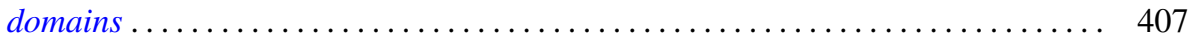

Stephen H. McCleary, The lattice-ordered group of automorphisms of an $\alpha$-set . . . 417

Stephen H. McCleary, o-2-transitive ordered permutation groups .......... 425

Stephen H. McCleary, o-primitive ordered permutation groups. II . . . . . . . . . 431

Richard Rochberg, Almost isometries of Banach spaces and moduli of planar

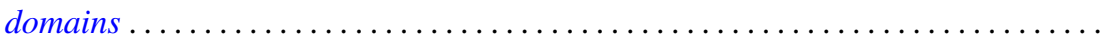

R. F. Rossa, Radical properties involving one-sided ideals . . . . . . . . . . . . . 467

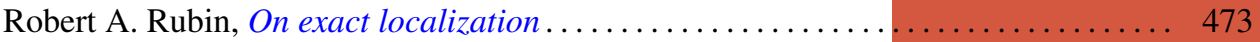

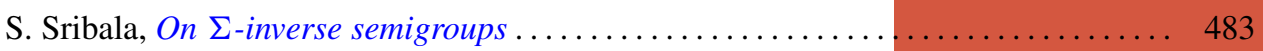

H. M. (Hari Mohan) Srivastava, On the Konhauser sets of biorthogonal polynomials suggested by the Laguerre polynomials ...................... 489

Stuart A. Steinberg, Rings of quotients of rings without nilpotent elements . ...... 493

Daniel Mullane Sunday, The self-equivalences of an $H$-space . . ............ 507

W. J. Thron and Richard Hawks Warren, On the lattice of proximities of $\check{C} e c h$

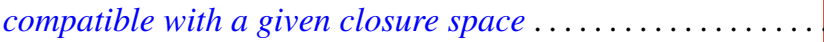

Frank Uhlig, The number of vectors jointly annihilated by two real quadratic forms determines the inertia of matrices in the associated pencil .

Frank Uhlig, On the maximal number of linearly independent real vectors annihilated simultaneously by two real quadratic forms ..............

Frank Uhlig, Definite and semidefinite matrices in a real symmetric matrix pencil . . 561

Arnold Lewis Villone, Self-adjoint extensions of symmetric differential operators . . . 569

Cary Webb, Tensor and direct products . ....................... 579

James Victor Whittaker, On normal subgroups of differentiable

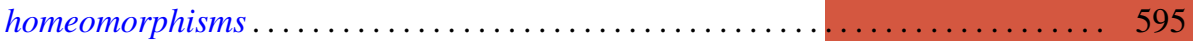

Jerome L. Paul, Addendum to: "Sequences of homeomorphisms which converge to

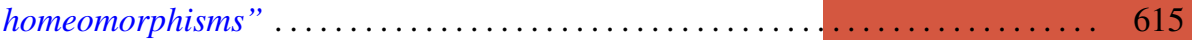

David E. Fields, Correction to: “Dimension theory in power series rings” ........ 616

Peter Michael Curran, Correction to: “Cohomology of finitely presented groups”. . . 617

Billy E. Rhoades, Correction to: “Commutants of some Hausdorff matrices” ...... 617

Charles W. Trigg, Corrections to: "Versum sequences in the binary system” ...... 619 